\title{
Autour de l'année 1047 : un acte de Guillaume, comte d'Arques, pour Fécamp (18 juillet 1047)
}

David Bates, Pierre Bauduin

\section{Citer ce document / Cite this document :}

Bates David, Bauduin Pierre. Autour de l'année 1047 : un acte de Guillaume, comte d'Arques, pour Fécamp (18 juillet 1047). In: Cahier des Annales de Normandie n`35, 2009. De part et d'autre de la Normandie médiévale. Recueil d'études en hommage à François Neveux. pp. 43-52;

doi : https://doi.org/10.3406/annor.2009.2529

https://www.persee.fr/doc/annor_0570-1600_2009_hos_35_1_2529

Fichier pdf généré le 16/07/2018 


\title{
AUTOUR DE L'ANNÉE 1047 : \\ UN ACTE DE GUILLAUME, COMTE D'ARQUES, POUR FÉCAMP (18 juillet 1047)
}

\author{
David BATRS

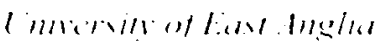 \\ Pierre B.ICDCIX

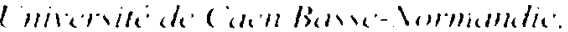

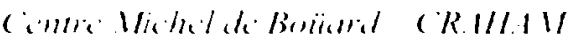

\begin{abstract}
" (ependamt. en 1046. (juillatume ke Batard an ait is ou 19) ans: il partenat a lage adule el semblait desormais en mesure de restaurer entin son pouroir dans le duches. Mais de nombreux seigneurs normands a aicm pris goult a l'anarchie. dont ils tiraient un maximum de

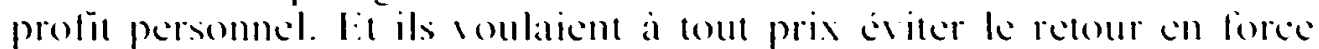
d'unc aturoriti, qui les controlerait. en contrariant leurs ambitions et ketrs interits".
\end{abstract}

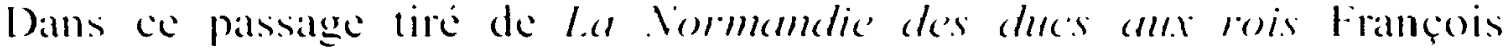
Neveux anmonçait lan des épisodes les plus lameux de l’histoire du duché. le "complot contre le dae" et la rébellion des grands de Vormandic yue

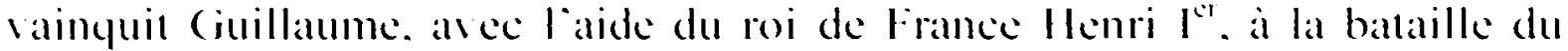

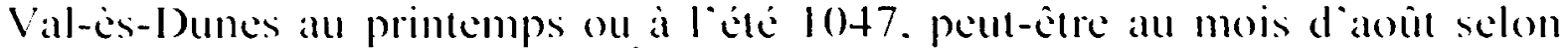
"unc tradition incontrobable ". Les latis onl depuis longemps suscite les commentaires de nombreux historiens". Note propos ne sera pas de presenter une nouvelle analyse de la période mais plus modestement de sortir de lombre un document yui. a sa maniere. éclaire le contexte de edte anmé cruciale du regne de (iuillaume le Batard. Il saggit d’une charte de ciuillaume, comte d'Arques ou de Talou daté du is juilled 1047 consertécen original au Muséc de la Bénédictine de Fécamp. dont nous proposons par ailleurs une édition assortic d"unc traduction".

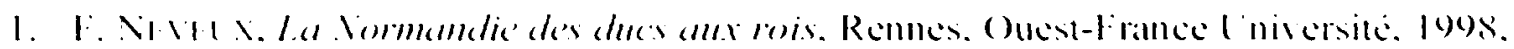
p. $111-112$.

2. Hick'm.p. 117 .

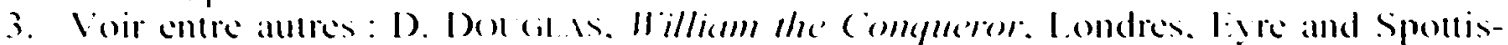

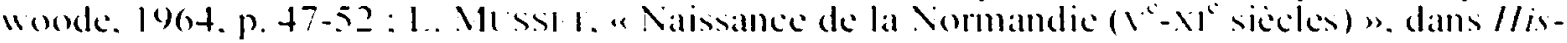

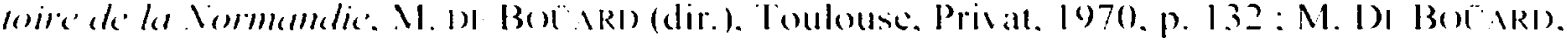

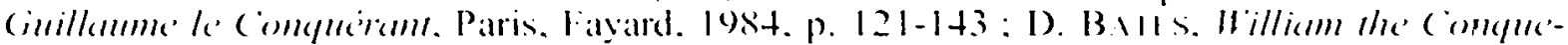

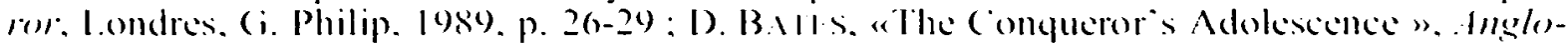

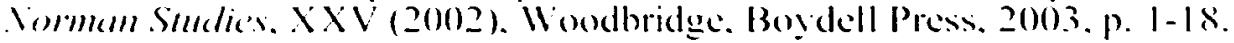

4. Votre recommaissance va ici a Marie-Agnes Lucas-Avemel ( $R A H A M)$ et ()lis icr Desbordes ( $(R A H A M)$ qui nous ont aimablement proposi cetle traduction yue nous al ons discu-

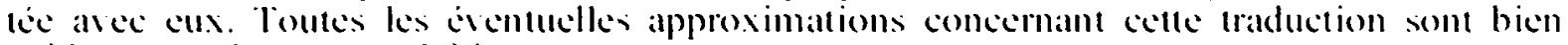
sidemment de notic seul fait. 
Laffaire est rapportéc par la charte et documentéc par au moins trois autres actes". dont le document a repris certaines formules. En 1025-1026. Renaud, vicomte d'Arques, av ait, aree l'aceord de Richard II, donné l'église et le domaine de Saint-Aubin-sur-Scie avec la terre de ee domaine et d'Appasilva. S'y ajoutaient un moulin. Tourville-la-Chapelle avec l'église et les dépendances. tout ee que Renaud possédait à Saintigny. à Arques el dans le comte d'Arques". (ette donation fut approuréc ensuite par Robert le Magnificue. duc de Normandic (1027-1035). Plus tard (1028-1035), par une come'nichnic élablic desant le due Robert et Robert. archevépue de Rouen (989-1037). Jean. abbé de Fécamp (1028-1078). aceordait la jouissance viagère de la terre cédéc par Renaud, à lexecption de Saintigny, à Goscelin tils d'Heddo (= (joseclin. vicome de d'Arques et de Rouen). à charge pour ee dernier de mettre en valeur eas biens yui devaient retourner dans le jus de labbaye apres la mort de Goseclin et de son épouse". Le domaine de Saintigny fut echangé ensuite par l'abbé avec une femme nomméc Emma. contre unc terre a Dunovilla". Un autre acte de Robert le Magnilique (rers 1031 1032-1035) nous apprend qu une partie des biens accordes par Renaud avait été usurpéc. puisque le due restitue à l'abbaye les terres concédés par Renaud à Arques. Tourville-la-Chapelle. Saintigny et dppasilial". (es biens avaient-ils été accaparés auparavant par Goscelin, auquel cas la comenientic pourrait navoir éte qu une régularisation de ectte situation ? Les documents ne lindiquent pas. en revanche la charte de (inillaume d' Arques montre que les relations entre l'abbé Jean et Goscelin furent tendues. Le vicomte. loin de respecter la coménienticr passéc arec l'abbé el de faire prospérer le bénéfice reçu - notons ici lemploi du mot beneficium qui renvoie ici clairement a une tenure viagere issue d"un accord contractucl ... "le ruina completement. et contrairement au contrat établi. il le répartit entre ses hommes ». (joscelin lit Egalement main basse sur la part que sétait résence labbe el la domna à son gendre. le vicomte ciodefroy. Face a cette situation lean el les moines de

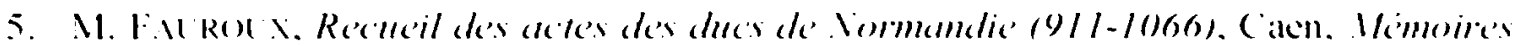

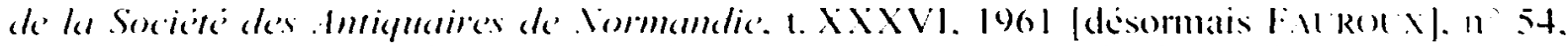

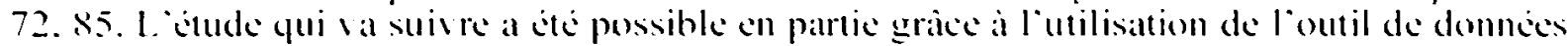
SCRIPTA (Site (alemnais de Recherche Intormaticue et de Publication des Textes Anciens) descloppe au (entre Michel de Boüard. (entre de recherches archeologigues et historiques

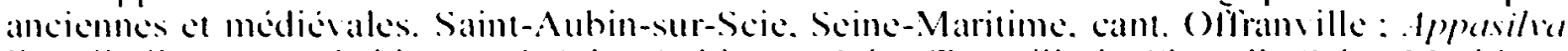
lieu-dit disparu. probablement a Sant-Subin-sur-Scie: Tour ille-la-chapelle Scinc-Maritime.

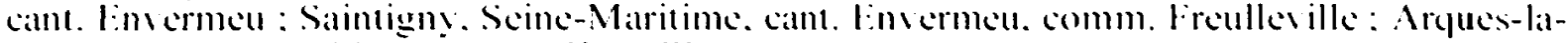
Bataille, Seinc-Maritime, cant. (Offranoille.

6. FNR $11 \times 11054$.

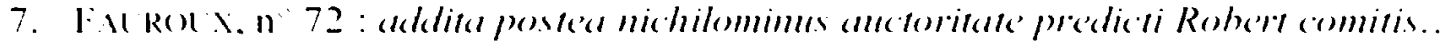

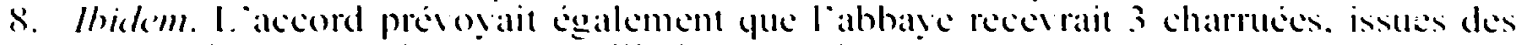
biens de (ioscelin. a (alleville (a Tourville-la-( hapelle).

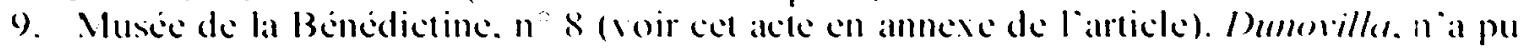
Etre identilic.

10. 1 : $1(x)$ X. n 5.5. 
Féamp intervinrent aupres de (iuilatume d'Arcues, afin qu il les remette en possession des biens usurpés. Avant dexaminer laation de (inillaume et ec qu elle nous suggere sur les prérogatives du comte en 1047. il nous faut considerer les acleurs de celle alfaire.

Il nest pas dans notre propos de revenir sur l"institution vicomtale". Renatud est le premier vicomte d'Arques attesté (en 1025-1026). il ne semble pas ètre en fonction atl-dela de cetle date. Son suceesseur. (ioscelin fils d'Heddo. est mieux connu mais datsantage comme vicomte de Rouen el fondateur de l'abbaye de la Trinile-du-Mont a Rouen (1030) ${ }^{12}$. Son titre vicomtal nest pas atteste pour Arques par des sources diplomatiques. mais par le

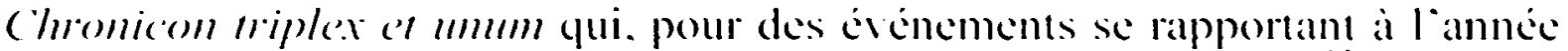

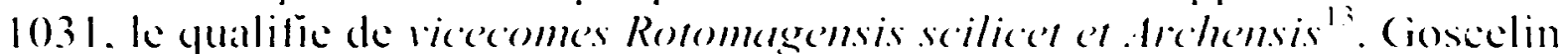
liut vieomte de Roten de 10301031 a une date comprise entre 1035 et 1045. annce ou il se retira a la Trinike-du-Mont el il mourut deux ans plus tard ${ }^{14}$. Il exerça la charge de vicomte d' Arques al ant 10.35. date vers layuelle son gendre el stecesseur. Ciodefroy. devint vicomte d'Arepues. Il est difticile détablir sil assuma ensemble les deux charges ou sil fut en fonction a Arques al ant (done vers $102526-1030)$ dexeres a Rouen. Quoi yüil en soit. comme le monte la charte de (juillaume d' Arques, (iosectin se montra particulierement entreprenant. ne reculant pas à consolider sa position en usurpant des biens d Figlise et en y installant des lideles ou des proches. Il fit également leun des premiers aristocrates normands apres le due a fonder une abbayes a la Trinite-du-Mont. a Rouen. Il est tres remarquable que (ioscelin n’hésita visiblement pas a adopter un modele princier voire royal . du moins si lon sen refere a l’une de ses chartes domnec a la Trinite-du-Mont vers 1030-1035. Dans cet acte souscrit par Robert le Magnificue. (ioscelin prend le titre bei gratia riceromes el son épouse bimmeline

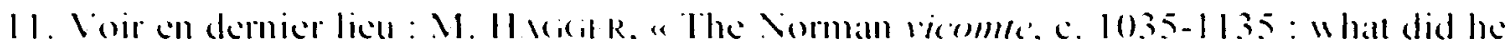

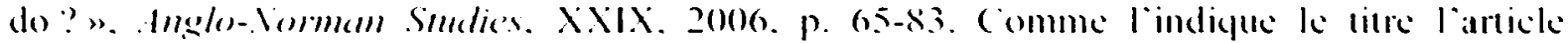
envisage exclusioment les fonctions du vicomte mais naborde pas la prosopographic de ess oflicicis.

12. I.- M. Bu VRIS. " Contribution a unc sude de l'institution vicomtale en Vormandic au

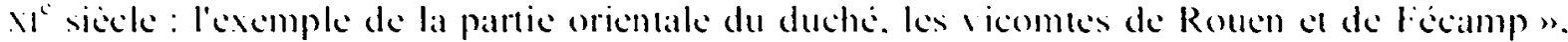

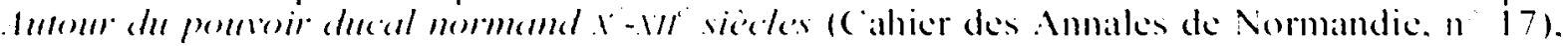

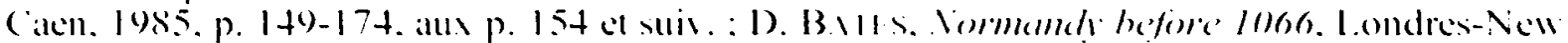

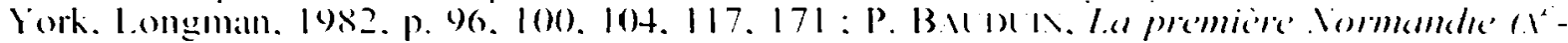

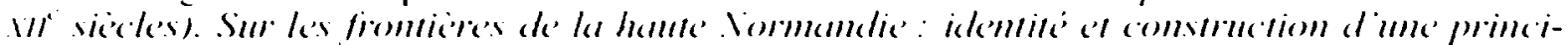

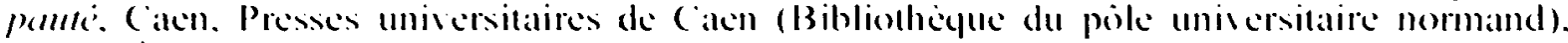
$2004(20$ idition. $20(0)$, p. 20$)$.

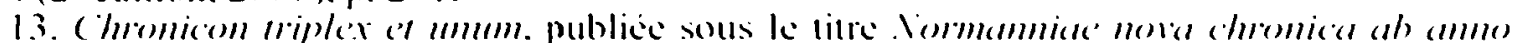

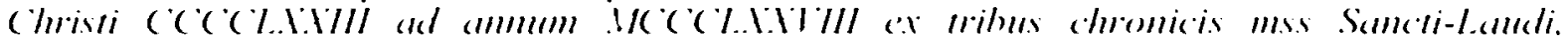

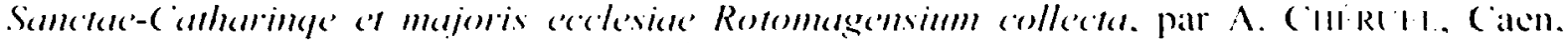

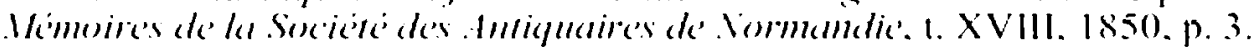

14. J.-M. Ba) VRIS. " Comtribution ..." "art. cit.. p. 155. 159. Sa mont est signalec par la charte de (juillaume d' Arques : elle sit donc antericure au le 18 juillet 1047.

15. J.-M. BOU TRIS. " Contribution..." ". art. cit.. p. 15.5. 
y apparait comme vicecemitissa ${ }^{10}$. Il manque encore une étude de la formule Dei gratia pour la Normandie. Celle-ci se diffuse a lechelon comtal en France aux $X^{\circ}-X^{\circ}$ siecles. sans toutefois se généraliser ${ }^{-}$. Pour les dues de Normandie, elle est attestée sous la forme grontic Dei (chw/Princeps). a partir de la fin du principat de Richard ${ }^{1 / .}$ et seulement depuis le regne de Cuillaume le Bâtard sous sa forme Dei graticl" : la charte de Goscelin présente donc l'une des toutes premières oceurrences pour la Normandie ${ }^{20}$ et demeure tress exceptionnelle pour un vicomte. Doit-on voir dans este affirmation de lorigine divine de lautorité vicomtale une remise en cause de la délégation du pouvoir ducal"? "est peu probable, car Goscelin soumet clairement son action a la reconnaissance du duc Robert (ammente domino meo comite silicet Rotherto $)^{21}$. Il est en revanche plus vaisemblable quen imitant le pouvoir princier. Goscelin cherche à attacher à sa fonction un surcroit de preslige. en la rehaussant au niveau de l'autorité exerée par le duc. ("est sans doute dans le même sens qu il faut interpréter la mention unique. à notre comnaissance, pour la Normandie du XI" siècle - de la "vicomtesse" Emmeline. La diffusion du titre de comirissa. depuis la lin du IX" siecte. témoigne a la fois de la transformation des structures du pouroir et de la parenté, où lépouse du comte se trouve mieux associéc au pouvoir de son mari et participe a la transmission héréditaire des honores. Régine le Jan a souligné que « le titre [de comitissa] est le pendant du e'go grotia bé comes qu il accompagne " : l"un comme l'autre marquant " la prétention des grands a exereer un pouvoir de mème nature que celui du roi et à y associer leur épouse ":2. Manifestement le processus est identique dans les années 1030 à léchelon vicomtal pour le cas de cioscelin. que l'on ne s'étonnera pas de voir fonder alors une abbaye (comme l’avaient fait jusque-la les dues) et

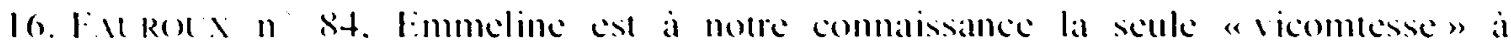
souscrire aimsi.

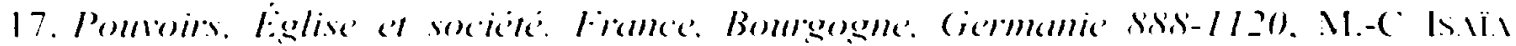
(dir.). Veuilly. Atlande. 2009. p. 185-186. Pour les iles Britanniyues apres 1060 voir

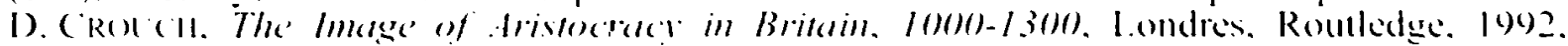
p. 13-14: la proliferation de es formules coüncide anec des periodes de lablesse pour le pousoir des rois, surtout en Angleterre pondant la periode de gutere cis ile entre le roi latienne et limperatrice Mathikds.

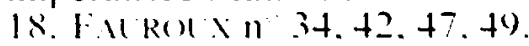

19. FIl Rol $\mathrm{x}$ n 109. 110. 111. Voir plus gencialement les remarques apportés par Maric Fauroux dans l introduction de som Recucil (p. +9).

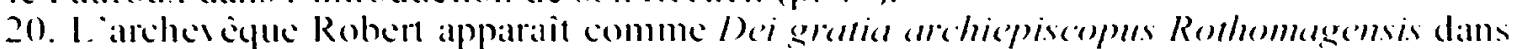

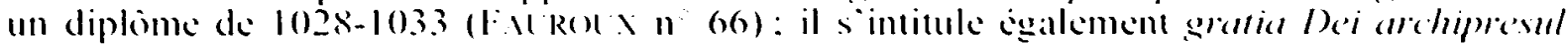

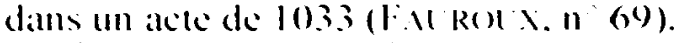

21. F $11 \mathrm{RO} \times 118 \mathrm{n}$.

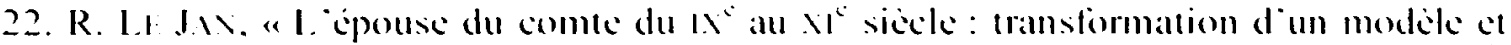

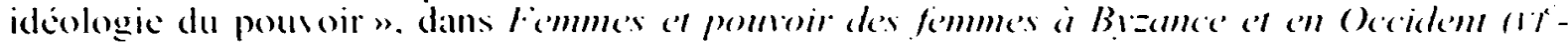

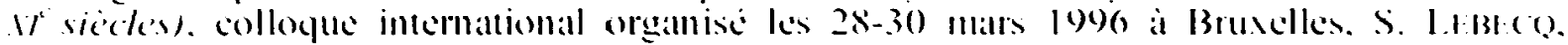

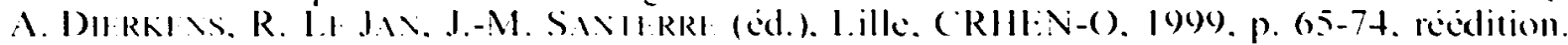

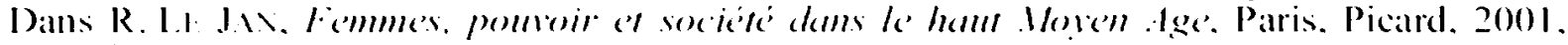
p. $21-29$ 
transmettre la charge vicomtale a l"un de ses proches. Il ne faut pas perde de

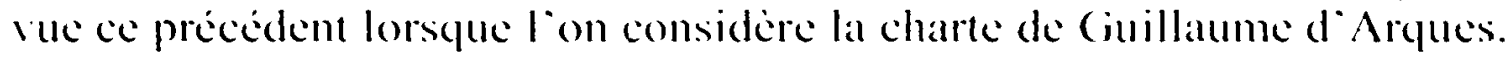

(iuillaume d'Arpues comme il se plait lui-même à le soulignere: était un lils de Richard II de Normandie el de Papie ${ }^{24}$. Fabli comme comte d'Areues par (iuillaume le Batard. peut-être des 10.37 el plus probablement all debul des amnés 1040. Le persomnage a suscike des eommentaires tres délarorables chey les chronicueurs du duché en raison de sa révolte contre le

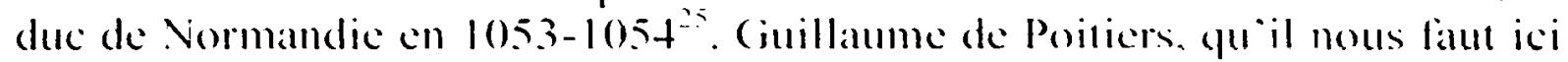
citer. insistait sur la déloyaute du comte bien arant sa révolte: "Par ses agissements sans nombre et sa turbulence. de longue date il arait tente d'aceroitre sa puissance el d'abaisser celle de son seigneur : a maintes reprises. il s'arma pour lui barrer l'aceés du chatcau d'Arques, roire même des eonlins de la Normandie en deça de la Seine. Finalement pendant le siege de Domfront. il s'eloigna fintivement. a la maniere d'un déserteur. sans aroir solliciti le conge. rejetant desormais le service vassalicye sous de courent ducuel jusqu'ici il a alit pu voiler en quelyue sorte son hostilite " $"$." I analyse de eses lémoignages el des ackes diplomatiques nous a incité récomment a aroir un jugement beatcoup plus nuance sur latetion de (iuillaume d' Ar-

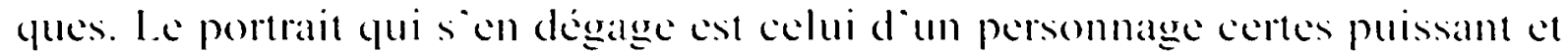
capable desendre son pouroir. mais aussi atlentil au maintien de leorde public dans le duché a la restauration des proprietes ecelésiasticpues el at rétablissement ou au renforecment de l’influence normande sur la fironticie orientak de la principaute. La charte de 1047 peut ainsi etre interprétéc de deux manieres selon que lon y roit le reflet de cette action positide ou une confirmation des soupçons que donne à lire le témoignagge de (iuillaume de Poitiers. Sa date (18 juillet 1047) ajoute une incertitude supplémentaire, car nous ne savons pas si a e moment (juillaume ke Bâtard avait ou non vaincu ses ennemis au Val-és-Dunes. (juillatume d'Arques agit sourerainement à la maniere du duc régnant. qui n’est nulle part cite. Mais saffranchit-il déjà

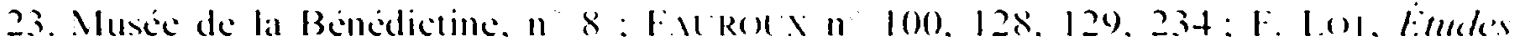

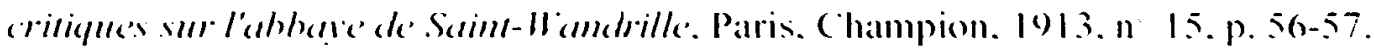

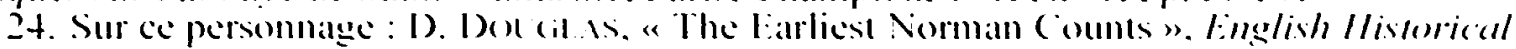

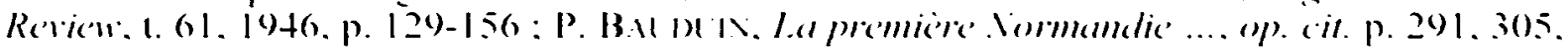

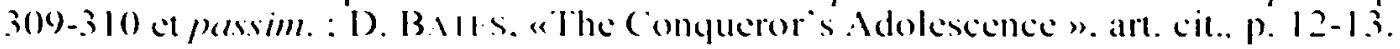

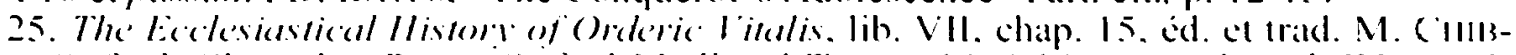

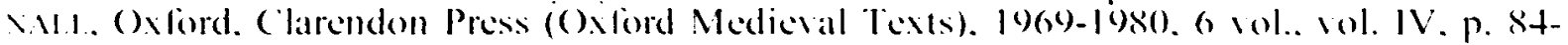

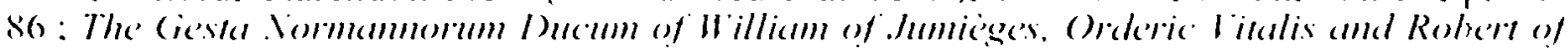

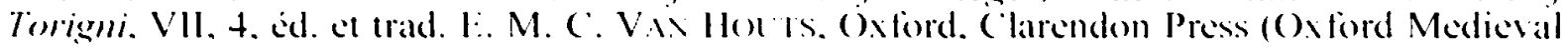
lix(s). 1992-1095. vol. 11. p. 102-104.

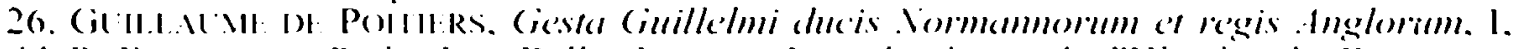

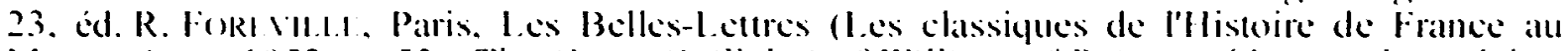
Moyen Age). 1952. p. 53: 7he (iesia (inillelmi of H'illiam of Poitiors, éd. et trad. anglatisc.

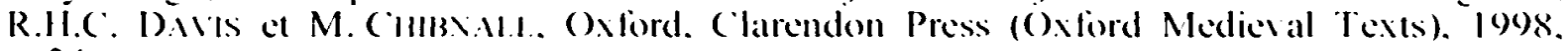
p. 34 . 
pour autant du pouroir ducal contre lequel il allait se rebeller ultéricurement?

La forme de l'acte s’inspire. pour partie du moins, d’un diplóme de Guillaume le Bâtard pour Fecamp daté de $1035-1040^{27}$. Le jeunc duc y apparait avec le titre bei gratic ...comes el cette aflirmation de lorigine divine du pouvoir est comme renforée en plaçant l'action du prince sous leffet de linspiration divine. Il est vaisemblable que l'adoption de ec formulaire pour la charte de Guillaume se lit à l’initiative de Fécamp. mais ce n est pas la premiere fois que le comte d'Arques reçoit une titulature inspiréce par celle des princes normands ${ }^{2 *}$. Il est probable. là encore. que lusage de ces litres vise à rehausser le prestige de (juillatume. Doit-on interpréter cette manifestation comme un geste d’indépendance a l'égard du pouvoir ducal ? Ce n'est pas ertain. La charte du comte d'Arques rappelle que (juillaume dut faire face aux «attaques iniques que mes emnemis araient dirigés contre moi " et que, par le passé. le vicomte Goscelin protita de la "jeune autorite " du comte pour semparer des biens de Féamp dans la région d Arques et y installer des fideles. Visiblement. Ie comte avait vu son pouroir conteste et arait dù affirmer son autorité sur le comté que lui arait accorde le duc Guillaume. Dés lors. doit-on interpréter laffirmation de sa superiorite fondée sur ses origines familiales et un pouroir tenu de Dieu - comme une réponse a cette situation? Le retrait puis la mort de (ioscelin et le contexte trouble de lannce 1047. poutaient justilier l'exaltation des prérogatives du comte sans qu il soit besoin d y voir là un prélude a la révolte du comte (iuillaume. Pour autant. il est diflicile de considérer cela uniquement comme le résultat de circonstances particulieres car notre personnage se trouve qualifie par d'atttres documents, notamment en provenance de l'abbaye de Saint-Wandrille. dans des termes yui soulignent sa position préminente aupres du duc ${ }^{20}$ et. plus génciralement. dans la lignec ducale. Lacte pour Fécamp. on le voit. documente une période diflicile de l'histoire de la Nomandie et une étape

27. F $\mathrm{Al} \mathrm{R}(\mathrm{x} \times \mathrm{n}$ "9): In nomine sancte et individua Trinitatis, ego Hillelmus gratia

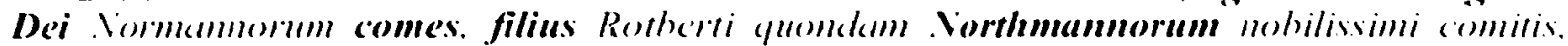

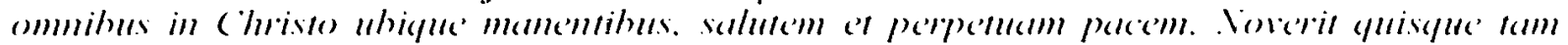

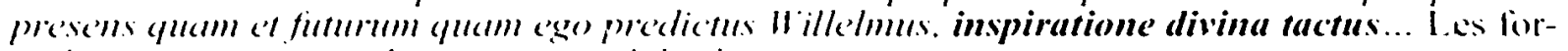
mules communcs aux deux actes ont éte mises en gras.

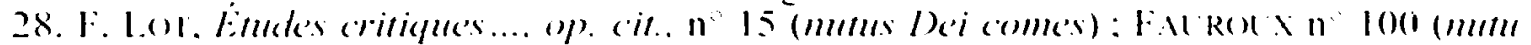

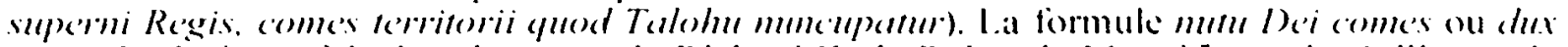
apparait plusicurs fois dans les actes de Richard II. de Robert le Magnificue, de (iuillaume ke Batard ou de keurs contemporains (ex. Yees. comte de Beaumont-sur-()ise). ("est également le cas pour larchereque Robert (mum l)ei archiepiscopms).

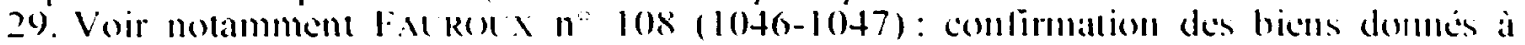

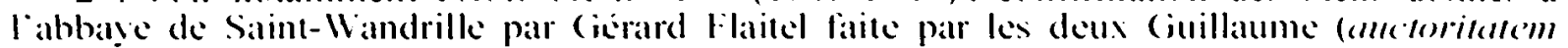

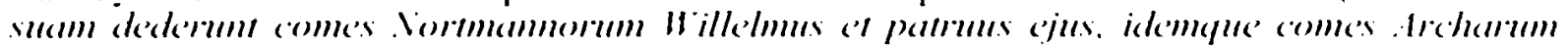

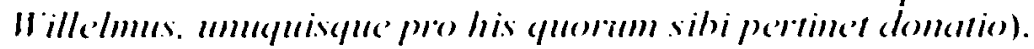


du rigne de (juillaume le Bâtard qui na pas encore live tous ses secrets aux historiens.

\section{Annexe \\ 1047. Is juilled Fécamp}

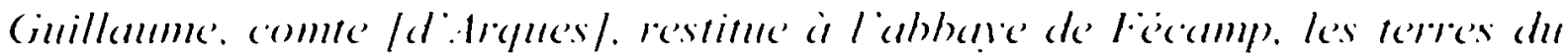

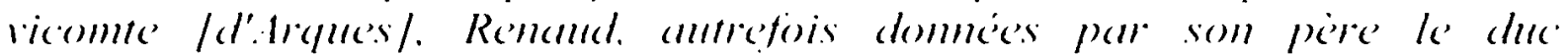

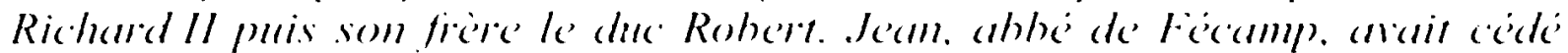

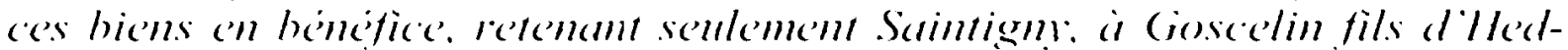

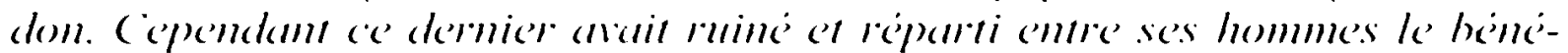

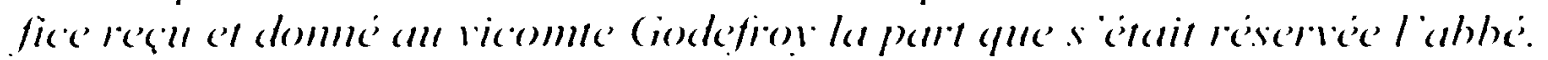

1. (Original. parchemin. $600605 \times 375.360$. dechire en hatu el en bals. liecamp. Musce de la Benedictinc. $n$ \& (ex. Lingel 5 bis) ".

B. Copic par dom J. Le Noir $1170+1$ Paris. Bnl: collection Morcau. wol. $3+1$. lol. 17-r. (d'apris . 1).

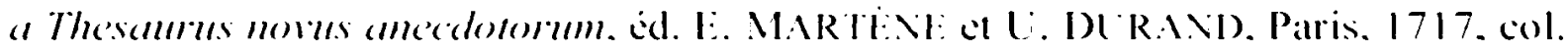
$160-168$.

ANAl Ysi: (ollection du Maryuis de Mathan. (hatcau de Semilly (Manche). manuscrits Dom L.enoir. vol. 70, p. 191.

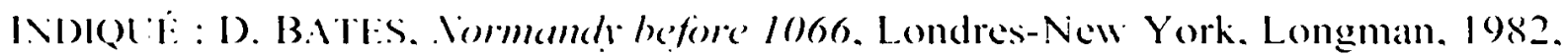

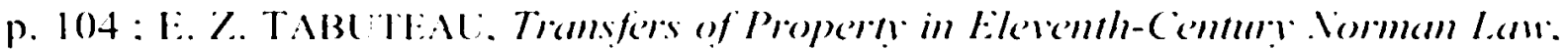
Chapel-llill and Londres. University of North California Press. 1988. n $8.32:$ l.a

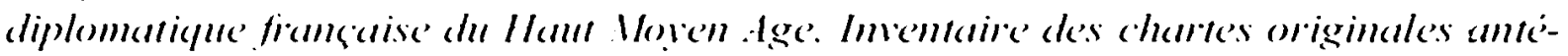
ricures a 1121 consemcies en Framce. B.-M. Tock (dir.). 2 vol. Turnhout. Brepols (Collection ARTI:M-4), 2001, n' 2697: D). BATI:S, "The (onqueror's Adolescence" ". Anglo-. Morman Shudies, XXV (2002). Woodbridge, Boydell Press, 2003. p. $1-18$ a a la p. 12.

30. Nous remercions 1'ARTIEM !Unisersité de Nancy 2) de nous avoir transmis une copic de la transcription réalisce pour la base de donnces des chartes originales anterieures a 1121 conserveses en france. 


\section{I) après A. complété par $B$ el a}

IN NOMINE PATRIS FT FILII IIT SPIRITLS SANCTI] |02] F(;O) WILLALMUS gratia Dei comes. Richardi secundi /Northmannorum / ducis lilius. inspiratione divina tactus. reminiseens propriorum actum $|0.3|$ admissa quat in adolescentia nimic perpetrat eram pariterque /pietatem] Dei expectantis me ad poenitentiam. ex intimo corde ai grates $[04 \mid$ innumeras juxta mei possibilitatem refero. qui mihi dedit velle |et possel derertendi a malo ad bonum. I:t ideo desidero ut omni populo |05| christiano innotescat ejus bonitas erga meam jusentutem |declarata. quac| non solum a lubricis actibus scparas it. verum eliam ab inimicis $|06|$ contra me inique insurgentibus mirabiliter protexit. Endique [eniml mihi lacpuess tetenderant. sed ipsis in cosdem corruentibus. ego liber [07] ab eorum insidiis Deo protegente inventus sum. Von atuem illis /gratiacl. sed salutis referantur atuctori. Quapropter volens recompensare fos| vicem beneficiis Dei. decrevi sanctam ejus aecelesiam honorare |servientes| ei sustentare et, si necessitas evenerit, pro cjus amore usque ad mortem $|09|$ ecrtare. Inter caeteras rero aceclesias, precipuc statui locum |liscannensem| in honote sanctac et individuac TRINITATIS consecratum mire $110 \mid$ attollere. et de bonis mihi a Deo coneessis remuncrare. el si qua |ab eol injuste ablata sunt quolibet modo per neglegentiam quarumeumgue $111 \mid$ personarum ex integro restituere. Votum sit igitur tam presentibus quam futuris quod pater meus Richardus comes dedit ommem lerram Rainaldi vice-112/-comitis monasterio fiscamnensi. ipso adhuc vivente yuam Rotbertus comes frater meus post cjus mortem comulit ex toto cum militibus. hospitibus. $|13|$ pratis. piscariis. molendinis. salinis. silvis omnive substantia. Postea vero abbas loci ipsius nomine Johannes largitus est ipsam terram (iomelino | $1+\mid$ Heddonis lilio loco benclicii. ut. quoad videret. teneret. possideret. pecudibus. porcariis. carrucis instrueres. et in sua dominicatura retineret. |15] nulli alio homini quicyuam de ipsa terra tribuens. Retinuit yuocpue predictus abbas de ipso alodo pro investitura villam yuate dicitur Santiniacus. 16 | 1 yuam poskea commutuas it " cuidam matronac nomine Emmae pro alia terra cuam ipsa tenchat in Dunovilla. eo yuod sibi vicinior haberetur. |17| Porro jamdictus (ioselinus. pars ipendens convenientiam cum abbatc en liatribus habitam. benclicium acceptum non solum non auxit. sed etiam ad nihilum $|18|$ redegit atcue suis hominibus contra statutam pactionem distribuit. Insuper namyue ipsam partem yuam sepefatus abbas sibi retinuctat. absc|ue ulla $119 /$ justitia Deicue timore de mea solummodo juenili potestate conlisus abstulit. et suo genero Godefiredo vicecomiti contradidit. Hace ratio [20] penc omnibus nota est. Inim vero. Deo precipiente. fincm vita sortitus est. recipiens justam retributionem factorum suorum. Accidit post haec |21| ut interpellarer a domno abbate Johanne ceterisque fratribus ipsius monasterii, quatinus memoratam hereditatem absque ulla cunctatione 122 in dominatione monachorum redire facerem. Quam interpellationem considerans justam esse et veram querimoniam necnon injustitiam [23] quam impie fierant perpessi, adepuievi voluntati ipsorum et. veniens in conventu corum cum quibusdam lidelibus meis, deliberas i sancta et individua |24| TRINITATI reddere "sepedictum alodum ad usus servorum cjus inibi communiter virentium. Hanc igitur donationem posui super altare sancti Salvatoris 125/ pro anima patris mei omniumque parentum meorum. necnon et pro mea anima et uxoris ac filiorum meorum. reddens Deo vero, trino et uno injuste subductam [26] possessionem a predecessoribus meis eollatam ex integro cum militibus. hospitibus. molendinis, piscariis. silvis, pratis. salinis omnicue suppel[27|-lectili, ut semper possideatur ab ipsius servis usque in acternum. [28] Si quis autem huic placito contrarius extilerit et alicuam molestiam loco fiscannensi pro 
ipsa re aliquo modo inferre templaterit. primitus |29| iram Dei incurrat et ab omnibus aceclesiac catholicae ordinibus excommunicetur. ac cum Juda traditore parkem habeal. si non digna $1.30 \mid$ sattisfactione emendaterit. Hujus autem comentionis lestes adficerunt hii : $131 \mid$ dommus abbas JOHANNIS el ommes sui monachi. Nicolaus etiam lilius Baldrici. Roubcrtus et Pontius litii Drogonis. 132| Aldulfus de

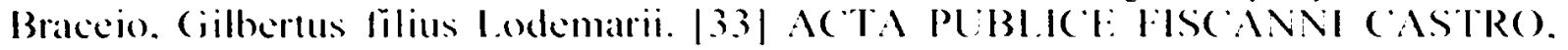

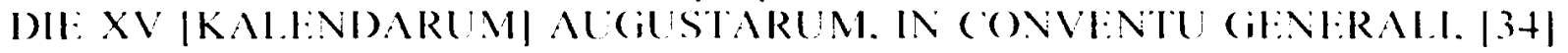
SUB PRIESIENTIA IOIIANNIS ABBATIS C AETIERORUMQLIE HRATRUM.

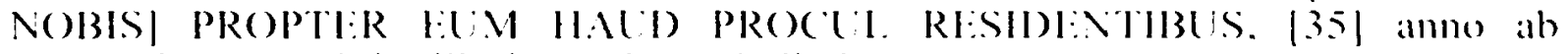
incarnations Domini millesimo Xl VII, indictions XV.

a) Sic. b) reddere en imerligns'.

\section{Traduction}

Au nom du Pere et du lils ed du Saint Reprit. Moi. (iuillaume combe par la grace de Dieu. tils de Richard II. due des Vormands. Euche par l'inspiration die ine me remsimorant les sants de conduite auxquels. dans ma prime jeunesse. je m'étas si souremt laissé aller. ainsi yue la miséricorde de Dieu. yui attend de moi le repentir. je lui rends grates infiniment. du fond du cocur. selon mes moyens. de matoir donne la volonte et la possibilite de me detourner du mal pour le bien. la c'est pourquoi. je desire que tout le peuple chrétien connatisie la bonté qu'il a manifestic enters moi durant ma jeunesse: non sculement it ma bloigne de mes penchants maurais. mais encore il ma protege miraculeusement des altayues in iques que mes ennemis avaient diriges contre moi. De toutes parts. en effet. ils mat aiem lendu

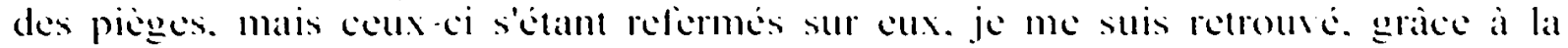
protection divine a l'abri de leurs machinations. (Grace en soit rendue non a eux. mais a l'atuteur de mon salut. ("st pourquoi. voulant reponde aux bienlats de Dieu par des actions de mème nature j'ai résolu d'honoter sa sainte liglise. de poursoir aux besoins de ses serviteurs el. en cas de necessile. de me battre par amour pour lui

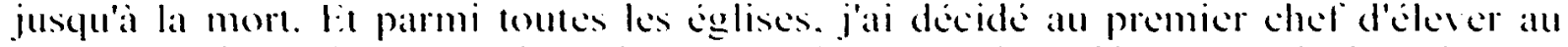
plus haut degré le monastire fde fecampl consalere en l'honneur de la sainte et indivisible Trinité. de la doter sur les biens yue Dieu m'a accordés el de lai laire restituer en totalité ec qui luil a elé injustement enlesé d'une maniere ou d'unc antre par la négligence de diverses personnes. In conséquence, que lous sachent. tant ceux presents qu à venir. que mon pere. le comle Richard. domna au monastie de Féamp toute la terre du vicomte Renaud. du vis ant de ce demier : qu'apres sa mort. le comte Robert. mon friere en lit donation en totalite aree ses chevaliers. ses hotes. ses pres. ses pêcheries. sés moulins. ses salines. ses forêts el toutes ses ressources pour viste. Mais ensuile l'abbe dudit monastere. Jean. fit don de celle terre a cioseelin. lils d Heddon. a titre de bénelice a charge pour lui. sa vie durant. de la tenir. posseder. pourvoir en bétail. en troupeaux de pores. en charrues. et de la maintenir dans sa reserve salns en rien éder a personne. De eed alleu. ledit abbé aussi garda en

31. Olivier Desbordes et Marie-Agnes L.ucas-Avellel. (entre Michel de Boüard (RAHAM. 
pleine propriéte le domaine de Saintigny ${ }^{2}$. qu'ensuite, arguant de sa proximite. il echangea al ec une dame du nom d'Emma contre une autre lerre que cette derniere detenait a Dunovilla" Seulement ledit (ioscelin. ne faisant aucun cas de la convention passéc arec l'abbe el les frères, loin de faire prosperer le bensfies reçu. le ruina completement et. contrairement au contrat élabli. il le répartit entre ses hommes. Qui plus est. presumant de ma jeunc autorite. il fit main basse. sans aucun esprit de justice ni crainte de Dieu. sur la partie que ledit abbe s'était réservée et il en fit don all vicomte (iodefiroy, son gendre. Presque tout le monde est al courant de ce litige. Il est vrai que. par la volonté de Dieu. il trouva la mort. receant ainsi la juste rétribution de ses actions. Apres ecla. il arriva que l'abbé. dom Jean. en tous les fireres dudit monastere me solliciterent. afin que je remette sans tarder les moines en possession du domaine en question. Ayant reconnu que leur sollicitation étail recevable. que leur reclamation élait fonde et qu'était ar eré l'injustice qu'ils araient eu à endurer sans l'avoir méritée. j'ai consenti à es yu'ils roulaient et. m'étant rendu en compagnie de plusieurs de mes fideles dans leur congregation. j'ai décide de la ire restituer l'alleu dont on parle à la sainte et indivisible Triniti. pour les besoins de ses serviteurs yui vivent là en communauté. J'ai donc déposé sur l'autel du saint Sauteur l'acte de cette donation, restituant au vai Died uniçe en trois persomnes, pour L le salut de l'âme de mon pere el celle de tous mes parents. ainsi que pour le salut de mon ame. eelle de mon épouse et edle de mes enfants. la possession. injustement volic. que mes prédicesseurs ar aient donnée en totalite aree ses chevaliers. hotes. moulins. pêcheries. forêts. prés. salines et tous ses produits domaniaux, afin que les serviteurs de cetle abbaye en soient proprictaires loujours et pour l'éternite. I:t si quelqu'un vient a contester celte resolution el a vouloir faire la-dessus. d'une maniere ou d'une autre. des enmuis a l'abbase de fecamp. qu'il encoure d'abord la coliere de Dien et soil excommunié par tous les dignitaires de l'íglise catholique. et yu'il partage le sort du traitre Judas. s'il ne s'amende pas en faisant penilence comme il se doit. Ont assiste a la redaction de cette convention en qualité de temoins. l'abbe dom Jean el tous ses moines. Nicolas fils Baudry" Robert et Pons fils de Dreux. Adoul de Brachy" (jilbert fils de l.odemer. Fait publiquement dans le chateau de lecamp. Ie 18 juillet. lors de l'assemblée genérale. en presenes de l'abbe Jän el de tous les frèes, et pendant que nous residions a proximite pour este assemblec, en l'an 1047 de l'inciarnation du Seigneur. indiction 15.

32. Scinc-Maritime. cant. Finsermen. comm. Freullerille.

3.3. L ieu-dit mon identitie.

34. Nicolas fils Baudry scigneur de Guitry. Stait issu d'un lignage bien implante en

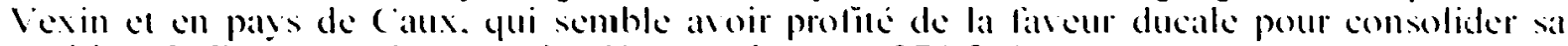

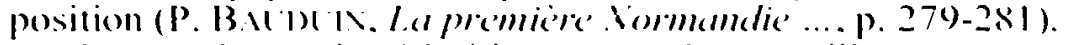

35. Brachs. Scine-Maritime. cant. Bakculeville-en-caus. Adoul de Brachs souscrit un

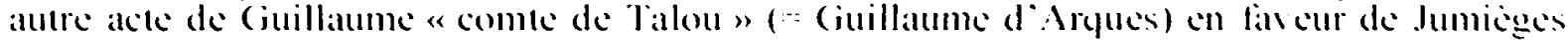

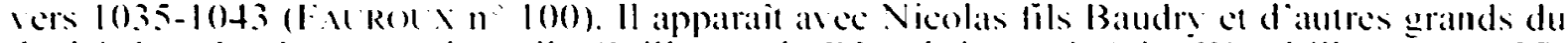

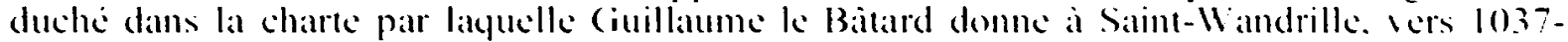

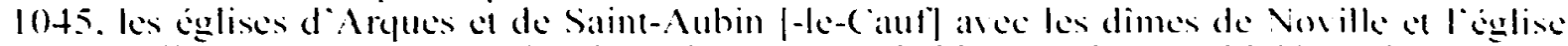

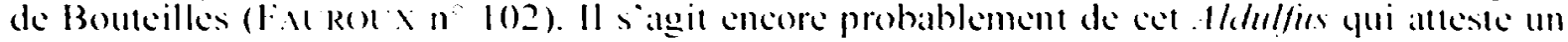
acte par leyuel Nicolas lils Bandry donne legglise de (iuitry au meme etablissemem entre

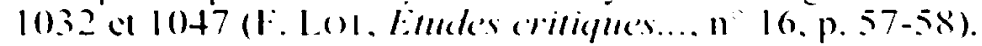

